\title{
Measurement of SARS-CoV-2 RNA in wastewater tracks community infection dynamics
}

\author{
Jordan Peccia $\mathbb{1}^{1,10 凶}$, Alessandro Zulli ${ }^{1,10}$, Doug E. Brackney ${ }^{2,10}$, Nathan D. Grubaugh $\mathbb{D}^{3}$, \\ Edward H. Kaplan (18,4,5, Arnau Casanovas-Massana ${ }^{3}{ }^{3}$, Albert I. Ko33, Amyn A. Malik ${ }^{6,7}$, \\ Dennis Wang6 ${ }^{6}$, Mike Wang ${ }^{6}{ }^{6}$, Joshua L. Warren ${ }^{8}$, Daniel M. Weinberger ${ }^{3}$, Wyatt Arnold ${ }^{1}$ and \\ Saad B. Omer (10) 3,6,7,9凶
}

We measured severe acute respiratory syndrome coronavirus 2 (SARS-CoV-2) RNA concentrations in primary sewage sludge in the New Haven, Connecticut, USA, metropolitan area during the Coronavirus Disease 2019 (COVID-19) outbreak in Spring 2020. SARS-CoV-2 RNA was detected throughout the more than 10-week study and, when adjusted for time lags, tracked the rise and fall of cases seen in SARS-CoV-2 clinical test results and local COVID-19 hospital admissions. Relative to these indicators, SARS-CoV-2 RNA concentrations in sludge were 0-2 d ahead of SARS-CoV-2 positive test results by date of specimen collection, 0-2 d ahead of the percentage of positive tests by date of specimen collection, 1-4 d ahead of local hospital admissions and 6-8 d ahead of SARS-CoV-2 positive test results by reporting date. Our data show the utility of viral RNA monitoring in municipal wastewater for SARS-CoV-2 infection surveillance at a population-wide level. In communities facing a delay between specimen collection and the reporting of test results, immediate wastewater results can provide considerable advance notice of infection dynamics.

The progression of the COVID-19 pandemic has been monitored primarily by testing symptomatic individuals for the presence of SARS-CoV-2 RNA and counting the number of positive tests over time ${ }^{1}$. However, in the United States and other countries, the spread of COVID-19 has commonly exceeded the testing capacity of public health systems. Moreover, test results are a lagging indicator of the pandemic's progression ${ }^{2,3}$, because testing is usually prompted by symptoms, which might take 2 weeks to present after infection ${ }^{4}$, and delays occur between the appearance of symptoms, testing and the reporting of test results. Monitoring sewage in a community's collection or treatment system has been used previously to provide early surveillance of disease prevalence at a population-wide level, notably for polio ${ }^{5,6}$, and might be similarly beneficial for the current COVID-19 pandemic. SARS-CoV-2 RNA is present in the stool of patients with COVID-19 (refs. ${ }^{7-9}$ ) and in raw wastewater ${ }^{10-12}$, and increased RNA concentrations in raw wastewater have been recently associated with increases in reported COVID-19 cases $^{11}$. However, the utility of wastewater SARS-CoV-2 concentrations for tracking the progression of COVID-19 infections is poorly understood. In this study, we investigated how viral RNA concentrations in
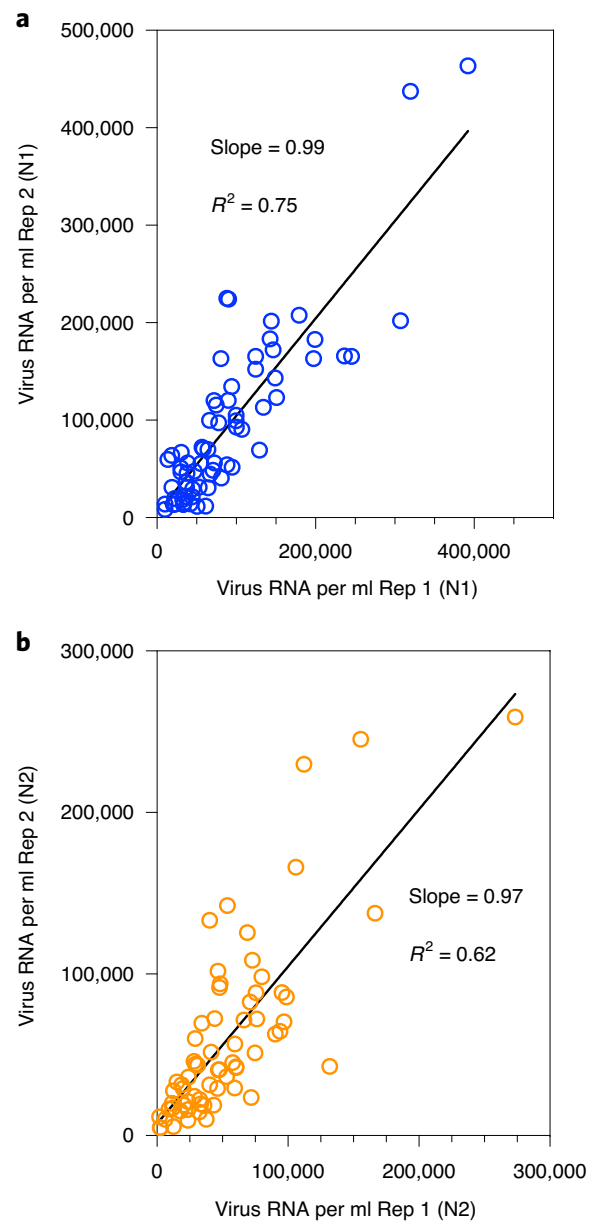

Fig. 1 | Replicate RNA extraction and analyses for SARS-CoV-2 RNA. a, Comparison of SARS-CoV-2 RNA concentration between two replicates (Rep 1 and Rep 2) using the N1 primer set. b, Comparison of SARS-CoV-2 RNA concentration between two replicates using the N2 primer set.

'Department of Chemical and Environmental Engineering, School of Engineering and Applied Science, Yale University, New Haven, CT, USA. ${ }^{2}$ Connecticut Agricultural Experimental Station, State of Connecticut, New Haven, CT, USA. ${ }^{3}$ Department of Epidemiology of Microbial Disease, School of Public Health, Yale University, New Haven, CT, USA. ${ }^{4}$ Public Health Modeling, Yale School of Public Health, New Haven, CT, USA. ${ }^{5}$ School of Management, Yale University, New Haven, CT, USA. ${ }^{6}$ Yale School of Medicine, New Haven, CT, USA. ${ }^{7}$ Yale Institute for Global Health, New Haven, CT, USA. ${ }^{8}$ Department of Biostatistics, Yale School of Public Health, Yale University, New Haven, CT, USA. ${ }^{9}$ Yale School of Nursing, Orange, CT, USA. ${ }^{10}$ These authors contributed equally: Jordan Peccia, Alessandro Zulli, Doug E. Brackney.凶e-mail: jordan.peccia@yale.edu; saad.omer@yale.edu 
a

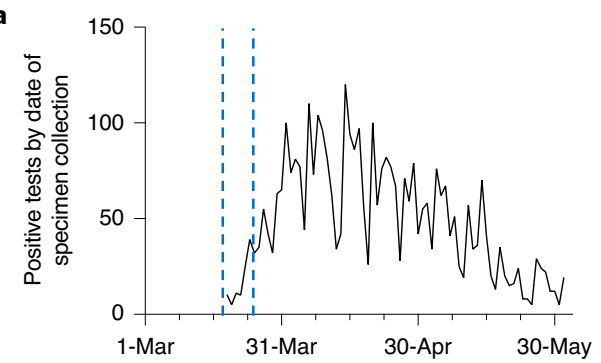

c

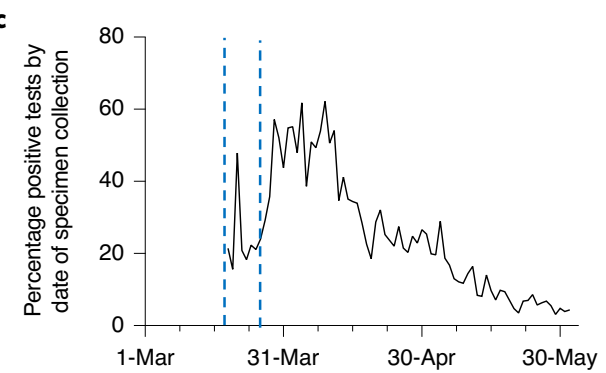

e

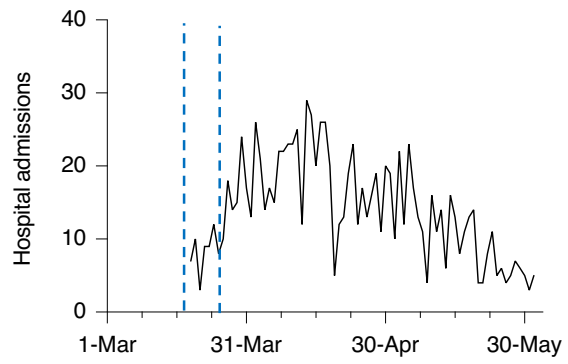

g

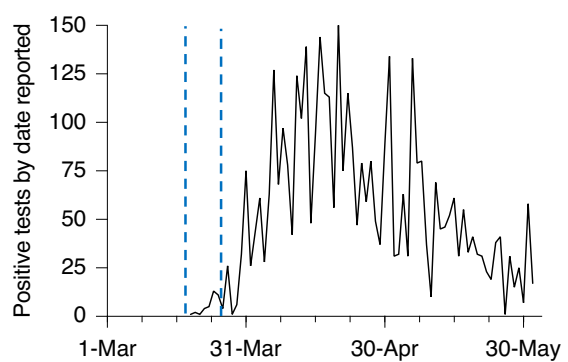

i

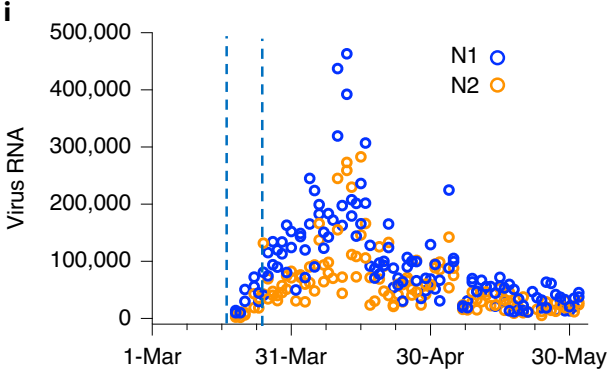

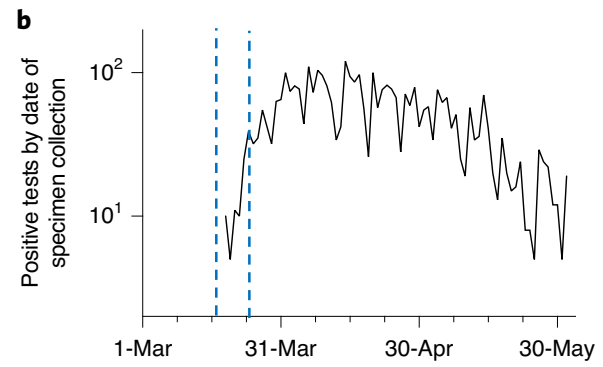

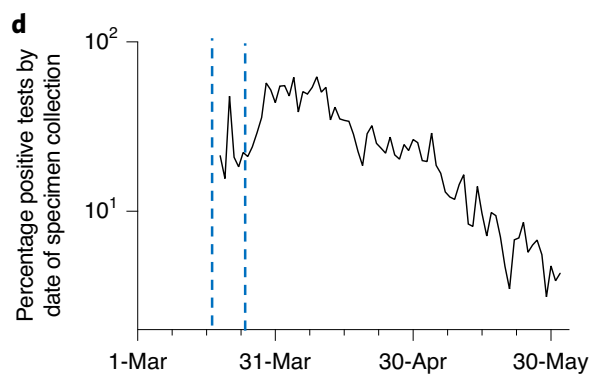

f

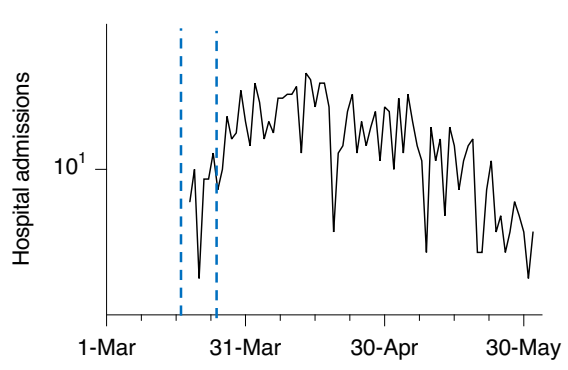

h

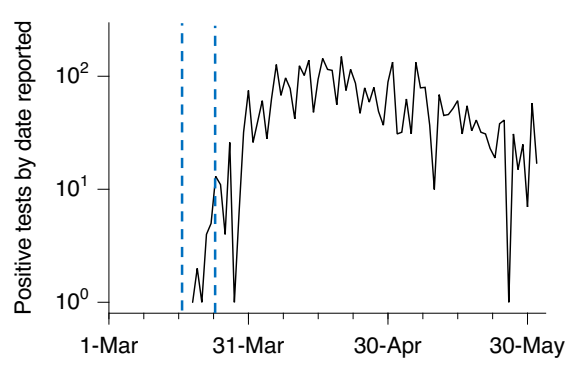

j

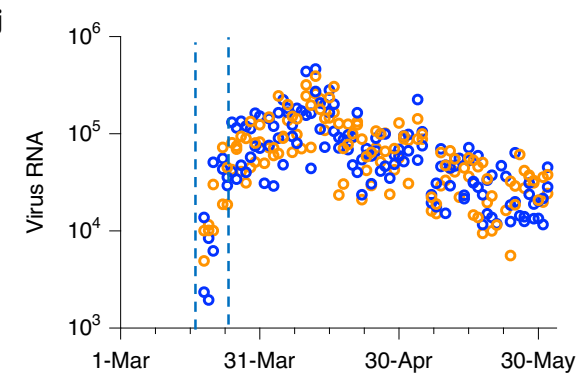

Fig. 2 | Sludge SARS-CoV-2 RNA concentration time course and other COVID-19 outbreak indicators on linear (left) and log (right) scales. All data represent the cities of New Haven, Hamden, East Haven and Woodbridge, Connecticut, which are served by the ESWPAF. The blue vertical dashed lines indicate the first week of analysis, March 19-25, 2020. a,b, Number of positive SARS-CoV-2 test results, reported by date of specimen collection. c,d, Percentage of positive SARS-CoV-2 test results, reported by date of specimen collection. e,f, Number of COVID-19 admissions to Yale New Haven Hospital for residents of the four cities. $\mathbf{g}, \mathbf{h}$, Number of positive SARS-CoV-2 test results by public reporting date. i,j, Primary sludge SARS-CoV-2 RNA concentration (virus RNA gene copies per ml of sludge).

wastewater correlated with compiled testing and hospitalization data in a U.S. metropolitan area over $\sim 10$ weeks, corresponding to a first wave of SARS-CoV-2 infection.
When municipal raw wastewater discharges into treatment facilities, solids are settled and collected into a matrix called primary sewage sludge. We chose to analyze primary sludge rather than raw 

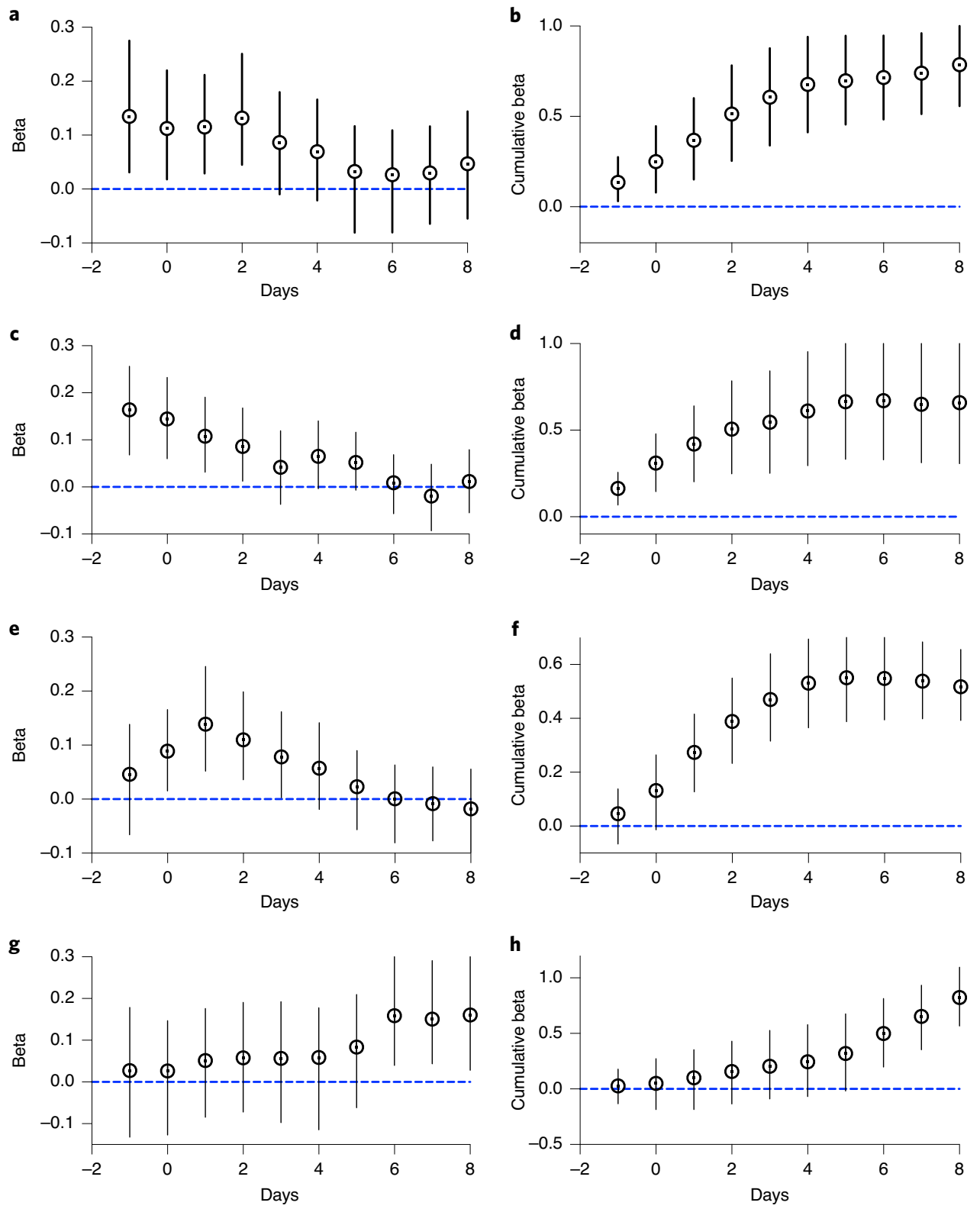

Fig. 3 Estimated daily distributed lag parameters describing the association between viral RNA in sludge and COVID-19 epidemiological parameters. a, Daily lags of 0-2 $d$ and leads of $1 \mathrm{~d}$ are associated with the number of positive tests based on specimen collection date. $\mathbf{b}$, Cumulative relationship between viral RNA in sludge and the number of positive tests based on specimen collection date. c, Daily lags of 0-2 d and leads of $1 \mathrm{~d}$ are associated with the percentage of positive tests based on specimen collection date. $\mathbf{d}$, Cumulative relationship between viral RNA in sludge and the percentage of positive tests based on specimen collection date. e, Daily lags of 1-4 d are associated with hospitalization. $\mathbf{f}$, Cumulative relationship between viral RNA in sludge and hospital admissions. g, Daily lags of sludge virus RNA data at longer time lags (6-8 d in the past) best correlate with the time series of publicly reported positive tests. $\mathbf{h}$, Cumulative beta relationship between viral RNA in sludge and reported number of positive tests. Posterior means at the center of each data point and $90 \%$ credible intervals for error bars are displayed. For each lag, $n=75$ daily values for positive tests by date of specimen collection $(\mathbf{a}, \mathbf{b}), 75$ daily values for percentage of positive tests by date of specimen collection $(\mathbf{c}, \mathbf{d}), 75$ daily values for hospital admission (e,f) and 75 daily values for publicly reported positive tests $(\mathbf{g}, \mathbf{h})$.

wastewater because it provides a high-solids-content, mixed sample that has been shown to contain a broad diversity of human viruses, including commonly circulating coronavirus strains $s^{13}$. During the COVID-19 outbreak, from March 19, 2020, to June 1, 2020, in the New Haven, Connecticut, metropolitan area, we collected daily primary sludge samples from the wastewater treatment facility, which serves $\sim 200,000$ residents. We quantitatively compared SARS-CoV-2 RNA concentrations in sludge with publicly reported data on four other measures of the outbreak: SARS-CoV-2 positive test results by date of specimen collection; the percentage of positive SARS-CoV-2 test results (test positivity) by date of specimen collection; the number of local hospital admissions of patients with COVID-19; and SARS-CoV-2 positive test results by reporting date.

We measured SARS-CoV-2 virus RNA by quantitative reverse transcription polymerase chain reaction (qRT-PCR) using the same $\mathrm{N} 1$ and N2 primer sets employed in COVID-19 individual testing. Virus RNA copies ranged from $1.7 \times 10^{3} \mathrm{ml}^{-1}$ to $4.6 \times 10^{5} \mathrm{ml}^{-1}$ of primary sludge. All qRT-PCR concentration threshold $(\mathrm{Ct})$ values 
were below 40, and $97 \%$ of all samples had a Ct value less than 38 . The average Ct was 34.6 for N1 primers and 34.5 for N2 primers. Values for each replicate were reported as positive only when the human ribonuclease $\mathrm{P}(R P)$ internal control gene was positive. The average (s.d.) Ct value for the $R P$ gene for all positive samples was 36.2 (1.2) for replicate 1 and 36.2 (1.3) for replicate 2. Replicated samples demonstrated similar SARS-CoV-2 RNA concentration values (Fig. 1). Concentration comparisons between replicates produced slopes of $0.99\left(R^{2}=0.75\right)$ for N1 primers and $0.97\left(R^{2}=0.62\right)$ for N2 primers.

All five measures traced the rise and fall of SARS-CoV-2 infections during the more than 10-week period studied (Fig. 2). However, the sludge results showed an increase during the first week (March 19-25, 2020) that was not observed in the reported testing or hospital admissions data. Applying a distributed lag measurement error time series model allowed an estimation of relationships between viral time series results and the reported testing and hospital admissions data. By modeling the epidemiological time series as a function of the sludge SARS-CoV-2 RNA data across multiple daily lags (posterior means $\pm 90 \%$ credible intervals), we found that the sludge results led the number of positive tests by date of specimen collection by $0-2 \mathrm{~d}$, with a potential lag of $1 \mathrm{~d}$ (Fig. 3a,b); the percentage of positive tests by date of specimen collection by $0-2 \mathrm{~d}$, with a potential lag of $1 \mathrm{~d}$ (Fig. 3c,d); hospital admissions by 1-4 d (Fig. 3e,f); and the number of positive tests by report date by $6-8$ d (Fig. 3g,h). Performing the time series analysis with or without adjustment for testing volume did not result in differences in estimated lag times between sludge viral RNA results and number of positive tests (based on the above date of specimen collection results and date reported to the Connecticut State Department of Public Health) (Extended Data Fig. 1).

Overall, our results demonstrate that measurement of SARSCoV-2 RNA concentrations in primary sludge provides an approach to estimate changes in COVID-19 prevalence on a population level. Sludge results were not a leading indicator compared to positive test results or percentage of positive tests by date of specimen collection. However, they led hospitalizations by 1-4 $\mathrm{d}$ and test results by report date by $\sim 1$ week. Thus, in communities where test reporting is delayed, sludge results, if analyzed and reported on the same day as sampling, can provide substantial advance notice of infection dynamics. In locations with rapid reporting of SARS-CoV-2 test results, the lead time afforded by sewage surveillance might be significantly reduced. The lags in test reporting have multiple causes and might vary with societal responses to the pandemic. COVID-19 arrived in the New Haven metropolitan area in early March 2020, when testing capacity was limited, and there were extended waiting times from test date to reporting date. Understanding and mitigating the causes of such lags will require additional research.

Sludge data are also susceptible to variability for multiple reasons. For example, primary sludge handling approaches are specific to particular treatment plants and could affect the levels of detectable virus. Given the uncertainties in sludge data and epidemiological data, we did not attempt to correlate absolute numbers of sludge SARS-CoV-2 RNA concentrations and COVID-19 cases.

Monitoring primary sludge is a broadly applicable strategy. Wastewater treatment plants with primary and secondary treatment are standard in many regions of the world, and treatment facilities are rapidly expanding in urban areas of lower- and middle-income countries $^{14}$. In the United States, approximately 16,000 treatment plants serve more than $250,000,000$ people. In regions without primary wastewater treatment, monitoring of raw wastewater streams would be necessary. Our results indicate that jurisdictions can use primary sludge SARS-CoV-2 concentrations as an additional basis for imposing or easing infection-control restrictions, especially in locations affected by limits in clinical testing capacity or delays in test reporting.

\section{Online content}

Any methods, additional references, Nature Research reporting summaries, source data, extended data, supplementary information, acknowledgements, peer review information; details of author contributions and competing interests; and statements of data and code availability are available at https://doi.org/10.1038/ s41587-020-0684-z.

Received: 17 May 2020; Accepted: 28 August 2020;

Published online: 18 September 2020

\section{References}

1. Dong, E., Du, H. \& Gardner, L. An interactive web-based dashboard to track COVID-19 in real time. Lancet Infect. Dis. 20, 533-534 (2020).

2. Mizumoto, K., Kagaya, K., Zarebski, A. \& Chowell, G. Estimating the asymptomatic proportion of coronavirus disease 2019 (COVID-19) cases on board the Diamond Princess cruise ship, Yokohama, Japan, 2020. Euro. Surveill. 25, 2000180 (2020).

3. García-Basteiro, A. L. et al. Monitoring the COVID-19 epidemic in the context of widespread local transmission. Lancet Respir. Med. 8, 440-442 (2020).

4. Lauer, S. A. et al. The incubation period of coronavirus disease 2019 (COVID-19) from publicly reported confirmed cases: estimation and application. Ann. Intern. Med. 172, 577-582 (2020).

5. Pöyry, T., Stenvik, M. \& Hovi, T. Viruses in sewage waters during and after a poliomyelitis outbreak and subsequent nationwide oral poliovirus vaccination campaign in Finland. Appl. Environ. Microbiol. 54, 371-374 (1988).

6. Berchenko, Y. et al. Estimation of polio infection prevalence from environmental surveillance data. Sci. Transl. Med. 9, eaaf6786 (2017).

7. Wang, W. et al. Detection of SARS-CoV-2 in different types of clinical specimens. JAMA 323, 1843-1844 (2020).

8. Xu, Y. et al. Characteristics of pediatric SARS-CoV-2 infection and potential evidence for persistent fecal viral shedding. Nat. Med. 26, 502-505 (2020).

9. Zhang, J., Wang, S. \& Xue, Y. Fecal specimen diagnosis 2019 novel coronavirus-infected pneumonia. J. Med. Virol. 92, 680-682 (2020).

10. Ahmed, W. et al. First confirmed detection of SARS-CoV-2 in untreated wastewater in Australia: a proof of concept for the wastewater surveillance of COVID-19 in the community. Sci. Total Environ. 728, 138764 (2020).

11. Medema, G., Heijnen, L., Elsinga, G., Italiaander, R. \& Brouwer, A. Presence of SARS-coronavirus-2 RNA in sewage and correlation with reported COVID-19 prevalence in the early stage of the epidemic in The Netherlands. Environ. Sci. Technol. Lett. 7, 511-516 (2020).

12. $\mathrm{Wu}, \mathrm{F}$. et al. SARS-CoV-2 titers in wastewater are higher than expected from clinically confirmed cases. mSystems 5, e00614-e00620 (2020).

13. Bibby, K. \& Peccia, J. Identification of viral pathogen diversity in sewage sludge by metagenome analysis. Environ. Sci. Technol. 47, 1945-1951 (2013)

14. Zhang, Q. H. et al. Current status of urban wastewater treatment plants in China. Environ. Int. 92-93, 11-22 (2016).

Publisher's note Springer Nature remains neutral with regard to jurisdictional claims in published maps and institutional affiliations.

(c) The Author(s), under exclusive licence to Springer Nature America, Inc. 2020 


\section{Methods}

Sample collection. Primary sewage sludge $(40 \mathrm{ml})$ was collected from the East Shore Water Pollution Abatement Facility (ESWPAF) in New Haven, Connecticut, USA. A total of 73 samples were taken daily from March 19, 2020, to June 1, 2020, between 8:00 and 10:00 EDT, and stored at $-80^{\circ} \mathrm{C}$ before analysis (samples were not available on May 3 and 6). The first sampling dates were before widespread testing in the region and before the March 23, 2020, stay-at-home restrictions implemented throughout the State of Connecticut. From the sampling start and end dates, cities served by the ESWPAF experienced an increase in confirmed COVID-19 cases (by testing) from seven cases to 3,978 (ref. ${ }^{15}$ ). The plant serves an estimated population of 200,000 people with average treated flows of $1.75 \mathrm{~m}^{3} \mathrm{~s}^{-1}$. Sludge collected from ESWPAF is primary sludge, sampled at the outlet of a gravity thickener, ranging in solids content from $2.6 \%$ to $5 \%$. The solids residence time in the gravity thickener is $4 \mathrm{~h}$

Viral RNA quantitative testing. To quantify SARS-CoV-2 RNA concentrations in primary sludge, $2.5 \mathrm{ml}$ of well-mixed sludge samples were added directly to a commercial kit optimized for isolation of total RNA from soil (RNeasey PowerSoil Total RNA Kit, Qiagen). Two replicate RNA extractions and analyses were performed for one daily primary sludge sample. Isolated RNA pellets were dissolved in $50 \mu \mathrm{l}$ of ribonuclease-free water, and total RNA was measured by spectrophotometry (NanoDrop, Thermo Fisher Scientific). SARS-CoV-2 RNA was quantified by one-step qRT-PCR using the U.S. Centers for Disease Control and Prevention (CDC) N1 and N2 primers sets ${ }^{16,17}$. For control and in accordance with the CDC protocol, analysis was also conducted for the human $R P$ gene $^{17}$, and SARS-CoV-2 results were reported only if $R P$ detection was positive. Samples were analyzed using the Bio-Rad iTaq Universal Probes One-Step Kit in 20 - $\mu \mathrm{l}$ reactions run at $50^{\circ} \mathrm{C}$ for $10 \mathrm{~min}$ and $95^{\circ} \mathrm{C}$ for $1 \mathrm{~min}$, followed by $40 \mathrm{cycles}$ of $95^{\circ} \mathrm{C}$ for $10 \mathrm{~s}$ and $60^{\circ} \mathrm{C}$ for $30 \mathrm{~s}$ per the manufacturer's recommendations. SARS-CoV-2 RNA concentrations were determined using a standard curve as previously described ${ }^{17}$ and presented as virus RNA copies. For the standard curve, complementary DNA synthesized from full-length SARS-CoV-2 RNA (WA1-USA strain) was used as a template to generate SARS CoV-2 $N$ gene transcripts as previously described ${ }^{17}$. The $N$ gene was amplified, and the PCR amplicon was purified and used for template in the MEGAscript T7 Kit (Thermo Fisher Scientific) to generate single-stranded RNA transcripts. RNA was quantified on a Qubit fluorimeter (Thermo Fisher Scientific) and integrity verified on a Bioanalyzer 2100 (Agilent). Viral RNA copies were calculated, and serial ten-fold dilutions were made. To validate our N1 and N2 primers sets, standard curves using the ten-fold series dilution $\left(5 \times 10^{1}\right.$ to $5 \times 10^{8}$ copies per reaction) of the $N$ gene transcripts were analyzed. The N1 primer set generated a standard curve with an $R^{2}$ value of 0.98 with an efficiency of $94.1 \%$ (slope $=-3.473 ; y$ intercept $=42.266$ ). The $\mathrm{N} 2$ primer set generated a standard curve with an $R^{2}$ value of 0.99 with an efficiency of $88.5 \%$ (slope $=-3.632$; $y$ intercept $=42.528$ )

The SARS-CoV-2 concentration results were adjusted to the total RNA extracted by multiplying sample concentrations by the ratio of the maximum RNA concentration to the sample RNA concentration. This accounts for day-to-day variations in sludge solids content and RNA extraction efficiency. To determine whether sludge RNA extracts contained PCR inhibition, target RNA was spiked into three separate sterile, ribonuclease-free water samples (no inhibition) and five different sludge RNA extracts from samples collected at a time in the outbreak when cases were low and viral RNA was not detected with N1 primers. Spiked samples were then diluted $5 \times$ and $25 \times$, and sludge RNA Ct values were compared to water RNA Ct values using N1 primers. No differences were observed for average water $\mathrm{Ct}$ values and sludge extract Ct vales for the no dilution $(P=0.14), 5 \times$ dilution $(P=0.51)$ and $25 \times$ dilution $(P=0.23)$, two-tailed $t$-test, suggesting no PCR inhibition in the RNA extracts. All samples were diluted $5 \times$ for use as a template to ensure that qRT-PCR inhibition occurred. Sewage sludge from March 2018 was used as a control, and no SARS-CoV-2 detection was observed from either N1 or N2 primers. These control sludges were stored at $-80^{\circ} \mathrm{C}$ and were consistently positive for the human $R P$ gene. Positive RNA controls and no-template controls were included in all qRT-PCR runs. Appropriate Ct values were observed for all positive controls, and no amplification was observed in negative template controls.

Epidemiological data. Daily COVID-19 admissions to the Yale New Haven Hospital were compiled from hospital records-adjusted to include only New Haven, East Haven, Hamden and Woodbridge, Connecticut residents-and confirmed by laboratory testing. Hospital data were obtained from the Joint Data Analytics Team for the New Haven Health System. The total number and percentage of tests of residents from the four cities that were positive for COVID-19 and reported by date of specimen collection and date of reporting to the State of Connecticut were supplied through a data request to the Connecticut Department of Public Health. Numbers of laboratory-confirmed positive COVID-19 tests by report date in the towns served by the ESWPAF (New Haven, East Haven, Hamden and Woodbridge, Connecticut) were compiled from daily reports published by the Connecticut Department of Public Health ${ }^{15}$.
Statistics. Linear regressions were used to estimate the relationship between SARS-CoV-2 RNA copies per ml results for replicated RNA extractions of each daily sample ( $n=73$ for each PCR primer). Two-tailed $t$-tests $(\alpha=0.05)$ were used in PCR inhibition experiments to determine if spiked sludge RNA extracts resulted in the same Ct values as spiked water samples at no dilution, $5 \times$ dilution and $25 \times$ dilution, $n=6$ for water spiked samples and $n=15$ for sludge spiked samples for each dilution.

Estimation of primary sludge as a potential leading indicator was performed using a distributed lag measurement error time series model. This analysis was carried out in the Bayesian framework, allowing us to correctly characterize multiple sources of uncertainty when estimating the lagged associations of interest. In the analyses, we assume that the observed sludge testing data represent unbiased estimates of an underlying, unobserved trajectory of viral concentration in the sludge. We then evaluated the association between the underlying trajectory of viral concentration in the sludge at multiple lagged periods and the number of positive tests (based on date of specimen collection)/percentage of positive tests based on date of specimen collection)/hospitalizations/number of positive tests (based on date of report) using distributed lag Poisson regression models that included a random effect to account for overdispersion and autocorrelation in the outcome, $n=73$ for sludge virus RNA samples, $n=75$ daily positive tests by date of specimen collection, $n=75$ daily percentage of positive tests reported by date of specimen collection, $n=75$ daily reported hospitalizations and $n=75$ daily reported positive tests. The distributed lag regression parameters were modeled using a random walk process. The models are fit using the rjags package in $\mathrm{R}^{18}$. Mathematical models include the following:

Model for case counts:

$$
\begin{gathered}
Y_{t} \mid \lambda_{t} \sim \operatorname{Poisson}\left(\lambda_{t}\right), t=d+1, \ldots, n-1 \\
\ln \left(\lambda_{t}\right)=O_{t}+\beta_{0}+\sum_{j=-1}^{d} \beta_{j} x_{t-j}+\phi_{t} \\
\phi_{t} \mid \alpha, \phi_{t-1}, \sigma_{\phi}^{2} \sim \operatorname{Normal}\left(\alpha \phi_{t-1}, \sigma_{\phi}^{2}\right) ; \phi_{0} \equiv 0
\end{gathered}
$$

Model for primary sludge:

$$
W_{t j} \sim \operatorname{Normal}\left(x_{t}, \sigma_{\epsilon}^{2}\right), \quad j=1, \ldots, m
$$

$$
x_{t} \sim \operatorname{Normal}\left(0,100^{2}\right)
$$

Prior distributions:

$$
\begin{gathered}
\beta_{0}, \mu \sim \operatorname{Normal}\left(0,100^{2}\right) \\
\beta_{j} \mid \beta_{j-1}, \sigma_{\beta}^{2} \sim \operatorname{Normal}\left(\beta_{\mathrm{j}-1}, \sigma_{\beta}^{2}\right), \mathrm{j}=2, \ldots, \mathrm{d} \\
\beta_{1} \sim \operatorname{Normal}\left(0,100^{2}\right) \\
\sigma_{\phi}, \sigma_{\epsilon}, \sigma_{\beta} \sim \operatorname{Uniform}(0,100) \\
\alpha \sim \operatorname{Uniform}(0,1)
\end{gathered}
$$

where $d$ is the number of past lags included in the model, $n$ is the total number of days of available data, $m$ is the number of primary sludge replicates on a given day, $Y_{t}$ is the case count on day $t, O_{t}$ is the offset on day $t$ (number of tests performed on each day for the analysis of cases by test date or 0 otherwise), $W_{t j}$ is the measured concentration of virus in sludge from sample replicate $j$ on day $t$, and $x_{t}$ is the unobserved true concentration of virus in sludge on day $t$.

Reporting Summary. Further information on research design is available in the Nature Research Reporting Summary linked to this article.

\section{Data availability}

The epidemiological data used in this study are provided as Source Data to Fig. 2. All sludge data are available upon request.

\section{References}

15. State of Connecticut, CT Data: Confirmed COVID-19 Cases. Connecticut Department of Health https://data.ct.gov/Health-and-Human-Services/ COVID-19-confirmed-cases-by-town-/28fr-iqnx (2020).

16. U.S. Centers for Disease Control and Prevention. CDC 2019-Novel Coronavirus (2019-nCoV) Real-Time RT-PCR Diagnostic Panel https://www. fda.gov/media/134922/download?fbclid=IwAR1DdEweazD3ixmrpZMc07V XM0_n1qX455rGV7E0fAEcA1QZf3Peh0Qxypo (2020). 
17. Vogels, C. B. F. et al. Analytical sensitivity and efficiency comparisons of SARS-CoV-2 RT-qPCR primer-probe sets. Nat. Microbiol. https://doi. org/10.1038/s41564-020-0761-6 (2020).

18. Plummer, M. JAGS: a program for analysis of Bayesian graphical models using gibbs sampling. In Proc. 3rd International Workshop on Distributed Statistical Comptuting (DSC, 2003).

\section{Acknowledgements}

We wish to thank the Greater New Haven Water Pollution Control Authority, New Haven, Connecticut, USA, for primary sludge sampling assistance. A.Z. is supported by a CoReCT seed grant from the Yale School of Medicine and the Yale Institute of Global Health. N.D.G. is supported by a gift from the Huffman Family Donor Advised Fund. A.K. and A.C.-M. are supported by the Beatrice Kleinberg Neuwirth Fund, the Yale School of Public Health and the National Institutes of Health (R01 AI121207). The authors thank K. Yousey-Hindes and P. Clogher for providing the data on percentage of positive tests.

\section{Author contributions}

J.P., A.Z., D.E.B., N.D.G., D.W., M.W. and W.A. contributed laboratory analysis and sample collection. J.P., E.H.K., A.C.-M., A.I.K., A.A.M., J.L.W., D.M.W. and S.B.O. contributed epidemiological analysis. D.M.W. and J.L.W. produced the distributed lag analysis.

\section{Competing interests}

The authors declare no competing interests.

\section{Additional information}

Extended data is available for this paper at https://doi.org/10.1038/s41587-020-0684-z. Supplementary information is available for this paper at https://doi.org/10.1038/ s41587-020-0684-z.

Correspondence and requests for materials should be addressed to J.P. or S.B.O. Reprints and permissions information is available at www.nature.com/reprints. 
LETTERS

NATURE BIOTECHNOLOGY

a

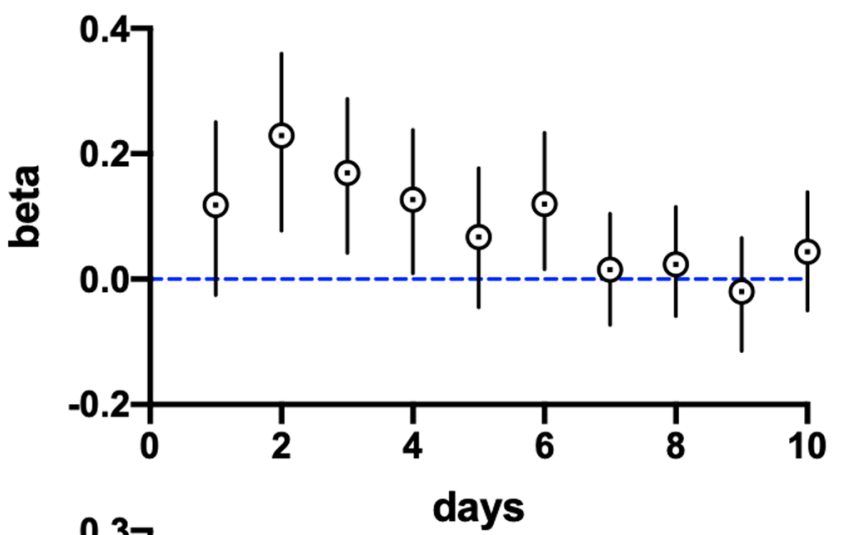

C

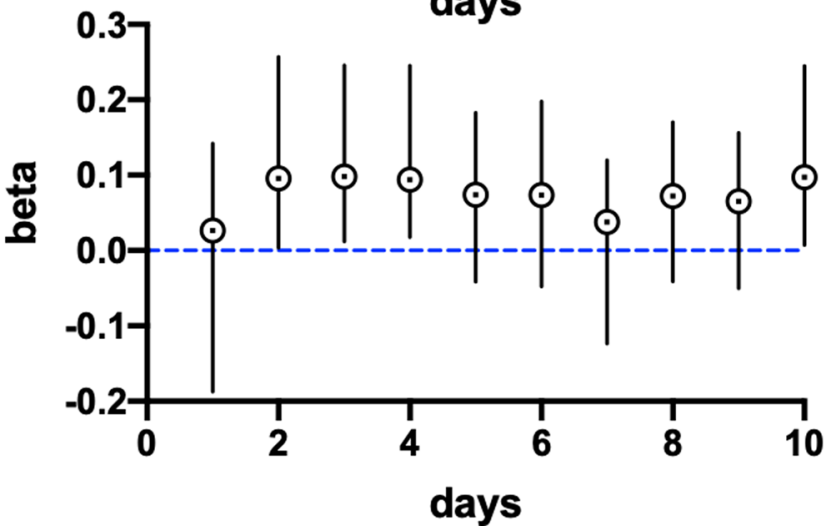

b

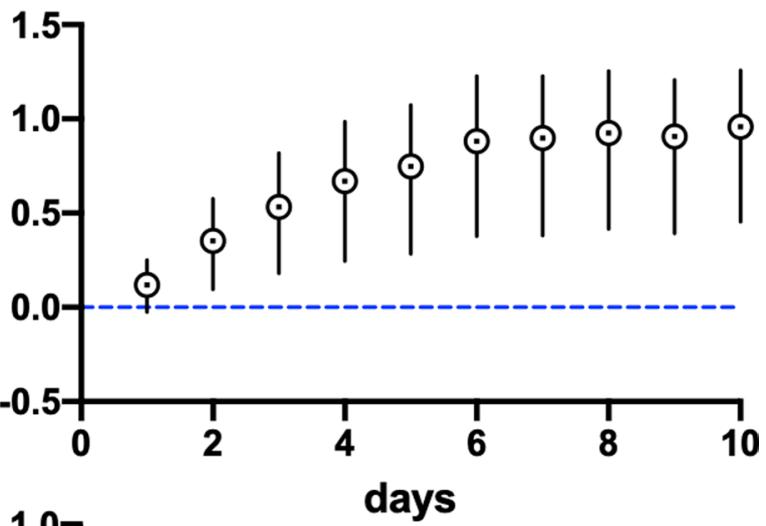

d

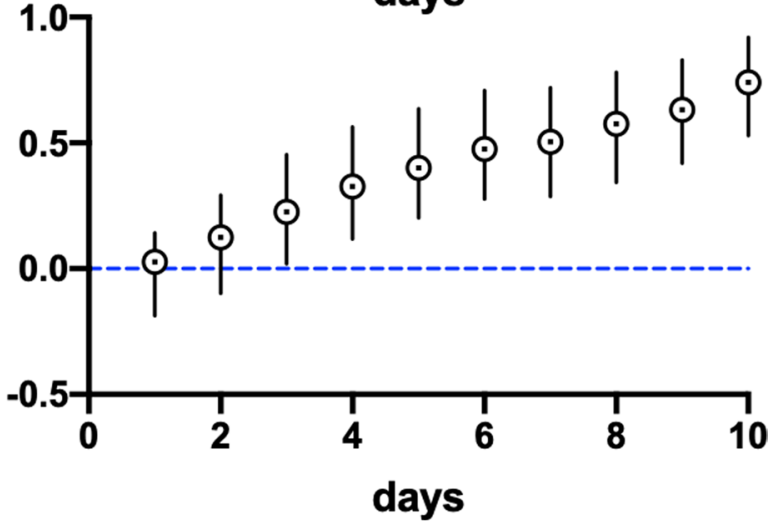

Extended Data Fig. 1 | Estimated daily distributed lag parameters describing the association between viral RNA in sludge and positive COVID-19 tests, based on date of report to CT Department of Health. The top row figures are adjusted for testing volume, bottom row figures are unadjusted for testing volume. a, daily lags of sludge data at longer time lags ( 0 to 4 days in the past) best correlate with the time series of reported positive tests (adjusted). b, cumulative relationship between viral RNA in sludge and number of positive tests (adjusted). c, daily lags of sludge data at longer time lags ( 0 to 4 days in the past) best correlate with the time series of reported cases (not adjusted for number of tests). d, cumulative relationship between viral RNA in sludge and number of positive tests (not adjusted). The posterior means at the center of each data point and $90 \%$ credible intervals for error bars are displayed. For each lag, $n=73$ daily virus RNA samples, $n=75$ daily reported adjusted positive test values, $n=75$ daily reported unadjusted positive test values.

NATURE BIOTECHNOLOGY | www.nature.com/naturebiotechnology 


\section{Reporting Summary}

Nature Research wishes to improve the reproducibility of the work that we publish. This form provides structure for consistency and transparency in reporting. For further information on Nature Research policies, see our Editorial Policies and the Editorial Policy Checklist.

\section{Statistics}

For all statistical analyses, confirm that the following items are present in the figure legend, table legend, main text, or Methods section.

n/a Confirmed

$\bigotimes$ The exact sample size $(n)$ for each experimental group/condition, given as a discrete number and unit of measurement

$\bigotimes$ A statement on whether measurements were taken from distinct samples or whether the same sample was measured repeatedly

The statistical test(s) used AND whether they are one- or two-sided

Only common tests should be described solely by name; describe more complex techniques in the Methods section.

$\bigotimes$ A description of all covariates tested

$\bigotimes$ A description of any assumptions or corrections, such as tests of normality and adjustment for multiple comparisons

$\square$ A full description of the statistical parameters including central tendency (e.g. means) or other basic estimates (e.g. regression coefficient)

AND variation (e.g. standard deviation) or associated estimates of uncertainty (e.g. confidence intervals)

$\varnothing$ For null hypothesis testing, the test statistic (e.g. $F, t, r$ ) with confidence intervals, effect sizes, degrees of freedom and $P$ value noted

Give P values as exact values whenever suitable.

\ For Bayesian analysis, information on the choice of priors and Markov chain Monte Carlo settings

\ For hierarchical and complex designs, identification of the appropriate level for tests and full reporting of outcomes

$\square$ Estimates of effect sizes (e.g. Cohen's $d$, Pearson's $r$ ), indicating how they were calculated

Our web collection on statistics for biologists contains articles on many of the points above.

\section{Software and code}

Policy information about availability of computer code

\section{Data collection no software was used}

Data analysis microsoft excel and $R$

For manuscripts utilizing custom algorithms or software that are central to the research but not yet described in published literature, software must be made available to editors and reviewers. We strongly encourage code deposition in a community repository (e.g. GitHub). See the Nature Research guidelines for submitting code \& software for further information.

\section{Data}

Policy information about availability of data

All manuscripts must include a data availability statement. This statement should provide the following information, where applicable:

- Accession codes, unique identifiers, or web links for publicly available datasets

- A list of figures that have associated raw data

- A description of any restrictions on data availability

COVID-19 new case data were obtained from publicly available data at the Connecticut Department of Public Health <https://data.ct.gov/stories/s/COVID-19-data/ wa3g-tfvc/>. Hospital admissions data was provided via request from the Yale New Haven Hospital Joint Data Analytic Team. The percentage of positive COVID-19 tests by date of specimen collection and by date reported to Connecticut Department of Health were supplied through a data request to the CT Department of Public Health. 


\section{Field-specific reporting}

Please select the one below that is the best fit for your research. If you are not sure, read the appropriate sections before making your selection.

$\square$ Life sciences $\quad \square$ Behavioural \& social sciences $\quad$ Ecological, evolutionary \& environmental sciences

For a reference copy of the document with all sections, see nature.com/documents/nr-reporting-summary-flat.pdf

\section{Ecological, evolutionary \& environmental sciences study design}

All studies must disclose on these points even when the disclosure is negative.

Study description

This study determined SARS-CoV-2 RNA concentrations in primary sewage sludge. These concentrations were taken each day for 73 days, during a COVID-19 outbreak. The time course of virus RNA in primary sewage was quantitatively compared to community epidemiological curves (based on testing) and hospital admission data and we showed that virus RNA concentrations were a leading indicator of outbreak dynamics.

Research sample

The research sample was primary sludge from a municipal wastewater treatment facility in New Haven, CT. Primary sludge was produced through primary sedimentation and gravity thickening.

Sampling strategy

Samples were collected leading up to the COVID-19 outbreak in New Haven, CT and ended as the outbreak had ended long after the outbreak ended (continued reduction in cases for `several weeks). Samples were collected daily.

Data collection

AZ, WA and JP extracted RNA and processed sludge, DB and NG conducted qRT-PCR. JP built data spreadsheets. EK, JW, DW led epidemiology analysis.

Timing and spatial scale

March 19 (before COVID-19 outbreak) to June 1 (near end of COVID-19 outbreak), samples daily for 75 days (samples were not available for 2 days in May).

Data exclusions

Data (less than 5\% with negative RP control genes were declared invalid in accordance with CDC prototocol

Reproducibility

Replicate RNA extractions were conducted on daily sludge samples, in addition two independent PCR primer sets were used for all analyses. Replicate RNA extractions were plotted to observe and quantify replicate relationships (Figure 1) .

Randomization

sample RNA extraction and analysis were performed in batches of 10 (5 samples, 2 replicates each). Dates were randomly chosen for each batch.

Blinding

Sampling at the gravity thickener was performed at the treatment facility employee, most RNA extraction performed by AZ, qRT-PCR performed by DB without knowledge of the sample dates or other information on expected content of each sample.

Did the study involve field work? \Yes $\square$ No

\section{Field work, collection and transport}

Field conditions

Wastewater treatment facility during the Spring of 2020, New Haven, CT.

Location

New Haven, CT, USA East Shore Water Pollution Abatement Facility

Access \& import/export

Permission obtained from facility manager and operator

Disturbance

none

\section{Reporting for specific materials, systems and methods}

We require information from authors about some types of materials, experimental systems and methods used in many studies. Here, indicate whether each material, system or method listed is relevant to your study. If you are not sure if a list item applies to your research, read the appropriate section before selecting a response. 
\ $\square$ Palaeontology and archaeology

$\triangle \square$ Animals and other organisms

$\bigotimes \square$ Human research participants

$\bigotimes \square$ Clinical data

$\square \square$ Dual use research of concern

\section{Dual use research of concern}

Policy information about dual use research of concern

\section{Hazards}

Could the accidental, deliberate or reckless misuse of agents or technologies generated in the work, or the application of information presented in the manuscript, pose a threat to:
No $\mid$ Yes
$\bigotimes \square$ Public health
$\bigotimes \square$ National security
Х Crops and/or livestock
\ $\square$ Ecosystems

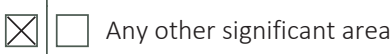

\section{Experiments of concern}

Does the work involve any of these experiments of concern:
No $\mid$ Yes
\ $\square$ Demonstrate how to render a vaccine ineffective
\ $\square$ Confer resistance to therapeutically useful antibiotics or antiviral agents
$\bigotimes \square$ Enhance the virulence of a pathogen or render a nonpathogen virulent
\ $\square$ Increase transmissibility of a pathogen
$\triangle \square$ Alter the host range of a pathogen
\ $\square$ Enable evasion of diagnostic/detection modalities
$\bigotimes \square$ Enable the weaponization of a biological agent or toxin
$\bigotimes \square$ Any other potentially harmful combination of experiments and agents 